\title{
3D bioprinting in microgravity: An end to organ donor shortages?
}

\section{Stephanie Yi Fei Lu ${ }^{1}$ and Adam M.R. Groh²}

${ }^{1}$ Division of Engineering Science, University of Toronto, Ontario, Canada

${ }^{2}$ Montreal Neurological Institute-Hospital, Department of Neurology and Neurosurgery, McGill University, Québec, Canada KEYWORDS: biofabrication, space, organ donation, 3D printing, ethics

\section{INTRODUCTION}

Countless lives have been saved with the advent of modern organ transplantation. However, the current shortage of compatible organ donors is limiting the life-saving potential of transplantation. According to the United Network for Organ Sharing, approximately 20 patients die each day in the United States while waiting for a transplant [1]. The discrepancy between supply and demand of organ donors is accentuated by a fundamental ethical dilemma associated with deceased organ donation: one person must die so that another may live [2]. The current viewpoint considers the viability of 3D bioprinting in microgravity as a solution to organ donor shortages. Current alternatives to deceased organ donation, including xenotransplantation and other state-of-the-art bioprinting techniques, are reviewed and compared to bioprinting in microgravity. The limitations of bioprinting within Earth's gravitational field are also discussed, revealing the need for further research.

\section{CURRENT SOLUTIONS}

One of the most readily available solutions to organ donor shortages is xenotransplantation, which is the crossspecies transfer of living cells, tissues, or organs. However, this method raises safety concerns considering the potential risk for transmission of zoonotic diseases [3]. Animals used for xenotransplantation must be quarantined and regularly screened for infectious diseases that threaten both the donor and recipient [3]. There also exists a negative social stigma surrounding xenotransplantation and evidence from a Swedish study suggests that only $40 \%$ of the general public are willing to accept an organ from an animal [4].

On the other hand, 3D biofabrication of organs provides a more ethically sound solution to organ donor shortages. Bioprinting is the simultaneous layer-by-layer stacking of biomaterials and living cells in a predetermined organization to fabricate organs and engineer tissues [5]. Extrusion-based bioprinting is the most broadly used bioprinting technology on Earth and it relies on forces created by pneumatic, mechanical, or solenoid-based systems to deposit bioinks in the form of continuous filaments [6]. Bioinks are materials that carry cells and are printed into scaffolds or tissue-like structures. In addition to producing structurally stable constructs, extrusion-based bioprinters address the issue of biological compatibility between donor organs and their recipients. These machines incorporate bioinks based on immunologically compatible materials such as platelet-rich plasma (PRP) [7]. PRP provides a patient-specific source of autologous growth factors that enhances angiogenesis (i.e., the process by which new blood vessels form from existing blood vessels), stem cell recruitment, and tissue regeneration, thus minimizing the chance of a host immune response after transplantation [7].

Not only do bioinks vary in composition and effectiveness, but different bioprinting methods utilize them in unique ways. Current state-of-the-art bioprinting methods include melt electrowriting, stereolithography, and laser-induced forward transfer [6, 8, 9]. Melt electrowriting deposits predefined micrometric fibres on a solid surface, building anatomically relevant 3D fibrous scaffolds for soft tissue engineering [8]. Stereolithography uses a digital light projector to selectively solidify a bioink in a layer-by-layer process, while maintaining high cell viability [9]. Laser-induced forward transfer utilizes laser power to directly transfer cells to the desired substrate through photopolymerization or cell-transfer based processes [6]. These advancements in 3D bioprinting are promising and offer unique solutions that do not necessitate death for associated transplantation. However, the biofabrication of structurally sound and complex geometries is still limited. 


\section{LIMITATIONS OF 3D BIOPRINTING}

Biofabrication of vascular networks is critical for organ function. However, achieving a desired level of cell density, effective vascularization, and accelerated tissue maturation are challenges that have not yet been overcome by bioprinting methods used within Earth's gravitational field [4]. Gravity threatens to collapse delicate 3D organs; without it, the threat is greatly minimized. Further, bioinks typically have a low viscosity at room temperature and solidify as they approach body temperature; structures bioprinted within Earth's gravity therefore need sufficient support to avoid collapse. Scaffolds can be used to support thick tissues, but they can also damage vascular networks and soft tissues. This makes gravity and temperature serious barriers to the use of 3D bioprinting technology as an alternative to transplants.

\section{BIOFABRICATION IN MICROGRAVITY}

Harnessing microgravity in space can help overcome bioprinting limitations posed by gravity. The main challenge to extrusion-based bioprinting on Earth is finding the optimal conditions between the printability and biocompatibility of the bioinks used. Printability is determined by parameters such as viscosity, surface tension, and the surface properties of the printer nozzle [10]. A viscous bioink will yield structures with higher stability and structural integrity; however, this high viscosity will require more pressure and force for successful extrusion - a process which may result in cell damage [10]. A biocompatible bioink must be non-toxic to other tissues and encourage bioactivity of cells after printing [10]. Earth's gravitational field favours bioinks with high viscosity because they maintain their 3D shape after deposition. However, viscous bioinks require the addition of chemicals and other materials to provide structural support. This compromises biocompatibility and permissiveness for post-printed sprouting angiogenesis, which is important to tissue vascularization [6]. Microgravity enables the use of finer-print tips and lower viscosity bioinks that contain only the biomaterials needed for a healthy organ, enabling high resolution bioprinting of more geometrically complex tissues with large empty spaces, channels, and voids such as the kidney and bladder [6]. These space-printed tissues can then be placed in a cell-culturing system to gain the strength required to withstand the pull of Earth's gravity [11]. Overall, geometrically complex organs that are otherwise challenging

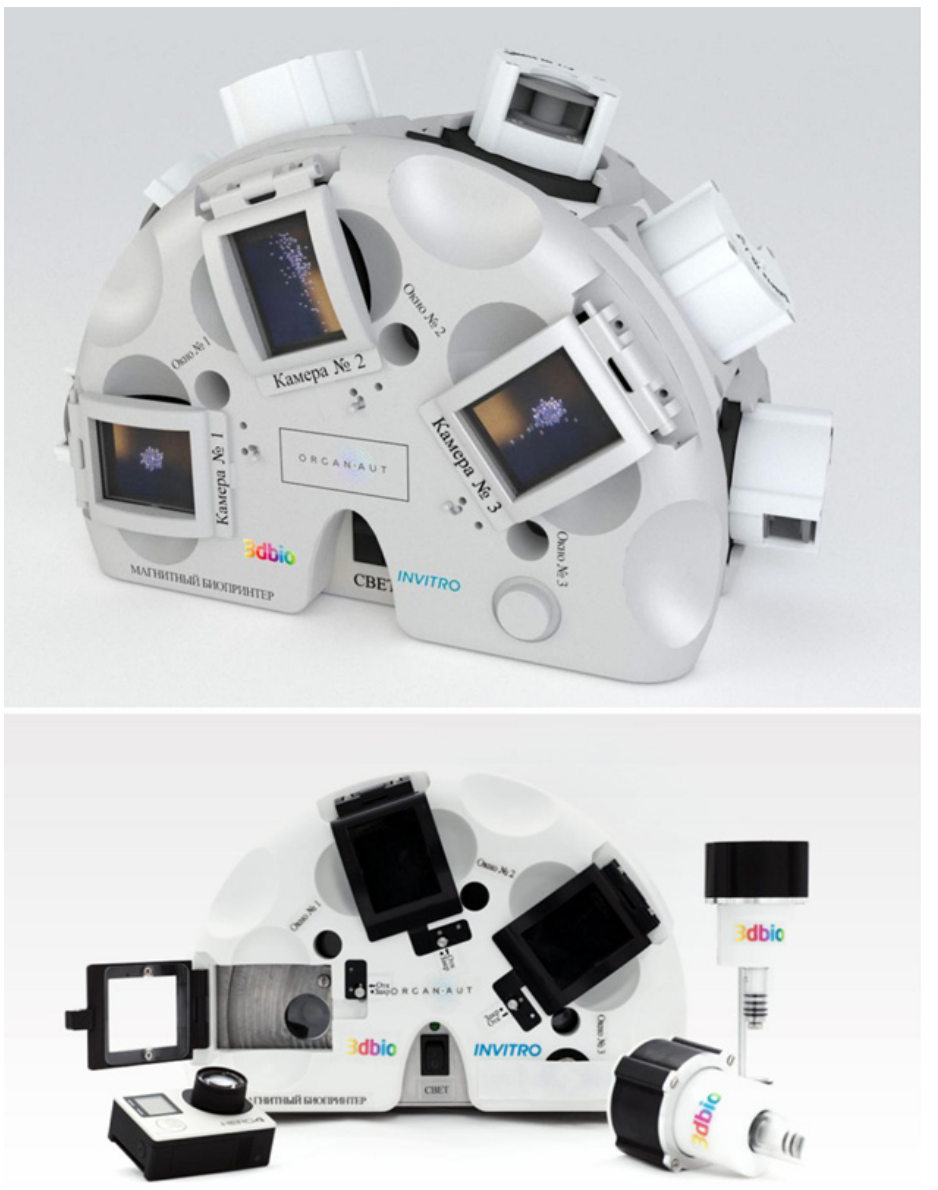

Figure 1. The design (top) and external view (bottom) of magnetic levitational bioassembler Organ.aut from 3D Bioprinting Solutions, Russia. In these designs, "Камера" refers to "camera" and "Окно" refers to "window". This figure was modified from Parfenov et al. [13], http://creativecommons.org/licenses/by/4.0/

to bioprint on Earth have the potential to be fabricated in the microgravity of space, rendering this novel technique a viable supplement to deceased organ donation.

Although extrusion-based bioprinters in microgravity enable the fabrication of more complex geometries, their slow speed will likely be unable to keep up with consumer expectation. Magnetic levitation is another emerging bioprinting technique that may help to overcome this issue. Russian company 3D Bioprinting Solutions has already developed a certified magnetic levitational bioassembler, Organ.aut (Figure 1), to perform bioprinting experiments in the International Space Station (ISS) as of October 2018 [10]. This technique utilizes a magnetic field that acts as a "scaffold" for organs, eliminating the need for traditional 
layer-by-layer bioprinting methods and potentially enabling programmed self-assembly [10]. This nozzle-free technique also avoids clogging problems, which would further slow the bioprinting process. Ultimately, this formative approach will enable biofabrication of complex tissue constructs directly in microgravity with high speed.

Magnetic levitation guides the assembly of diamagnetic objects such as cells or tissue spheroids into 3D constructs by using the magnetic field gradient generated by strong magnets in a paramagnetic medium (i.e., gadolinium) [12]. Unfortunately, gadolinium causes cytotoxicity and osmotic pressure imbalance when used in high concentrations. These adverse effects are undesirable to the biofabrication of immunologically compatible organs for transplantation [12]. However, in microgravity, bioassembly is possible using a paramagnetic medium with a one hundred times lower concentration of gadolinium, since a much smaller magnetic force is required to counteract the effects of gravitational acceleration [10].

Organ.aut was recently able to biofabricate the world's first 3D tissue-engineered constructs in space under microgravity; this experiment aboard the ISS used living tissue spheroids consisting of human chondrocytes [12]. Experimentation in space is still in its early stages, making it difficult to compare bioprinted constructs on Earth with those in the microgravity of space. Nevertheless, it is worth noting that the bioassembly and sequential fusion of the tissue spheroids created 3D constructs that had strong viability and reached advanced stages of tissue spheroid fusion [12]. Organ.aut's initial success suggests that there should be more experimentation with geometrically complex structures for a more thorough evaluation of bioprinting in microgravity as a solution to organ donor shortages.

\section{NEXT STEPS}

Despite the success and innovative potential of bioprinting in microgravity, bioprinted organs will no doubt raise new ethical questions and be subject to strict regulations. For example, if biofabrication is regarded as a profitable and patentable technology, will it be too expensive and inaccessible [4]? The ownership of bioprinted organs needs to be determined as well to ensure patient autonomy; if a patient receives an organ owned by a biotechnology company, how much power does the company have over the patient's body? Further, the rights and responsibilities of physicians, researchers, and biotechnology companies need to be clarified to prevent exploitation. A joint regulation between the legal and medical professions should be established to maximize the life-saving potential of bioprinted organs in microgravity. The ideal space bioprinter would also be userfriendly; advancements in automation could help astronauts bioprint organs in space in the absence of experts [10].

Organ.aut is scheduled to remain on the Russian segment of the ISS for at least 5 years, allowing the possibility for future experiments while substantially reducing shipping costs [12]. Furthermore, despite its compactness, the bioassembler can simultaneously perform six experiments, producing reproducible and statistically significant data [12]. This addresses one severe barrier to long-term experimentation in space: the cost of launching materials and re-supply [14].

\section{CONCLUSION}

Biofabrication in microgravity is an emerging innovation that has the potential to provide an ethically robust and technologically promising solution to organ donor shortages. The ability to employ more fluidic bioinks and the possibility to print more complex organ geometries make biofabrication in microgravity an exciting technological breakthrough. While the implementation of this technique may pose new questions, it has the capacity to revolutionize human health and build the foundation for an entirely new era of biological treatment and innovation.

\section{ACKNOWLEDGEMENTS}

I would like to thank STEM Fellowship for the opportunity to participate in the 2020 STEMpowerment Viewpoint Competition.

\section{REFERENCES}

1. Messner F, Guo Y, Etra JW, Brandacher G. Emerging technologies in organ preservation, tissue engineering and regenerative medicine: a blessing or curse for transplantation? Transpl Int. 2019 Mar 28;32(7):673-85. doi: 10.1111/tri.13432

2. Robba C, Fossi F, Citerio G. Organ donation: from diagnosis to transplant. Curr Opin Anaesthesio. 2020 Apr;33(2):146-55. doi: 10.1097/ACO.0000000000000826

3. Goerlich CE, Chan JL, Mohiuddin MM. Regulatory barriers to xenotransplantation. Curr Opin Organ Transplant. 2019 Oct;24(5):522-6. doi: 10.1097/ 


\section{MOT.0000000000000678}

4. Vermeulen N, Haddow G, Seymour T, Faulkner-Jones A, Shu W. 3D bioprint me: a socioethical view of bioprinting human organs and tissues. J Med Ethics. 2017 Mar 20;43(9):618-24. doi: 10.1136/medethics-2015-103347

5. Matai I, Kaur G, Seyedsalehi A, McClinton A, Laurencin CT. Progress in 3D bioprinting technology for tissue/ organ regenerative engineering. Biomaterials. 2020 Jan;226:119536. doi: 10.1016/j.biomaterials.2019.119536

6. Sun W, Starly B, Daly A, Burdick J, Groll J, Skeldon G, et al. The bioprinting roadmap. Biofabrication. $2020 \mathrm{Feb}$ 7;12(2):022002. doi: 10.1088/1758-5090/ab5158

7. Faramarzi N, Yazdi IK, Nabavinia M, Gemma A, Fanelli A, Caizzone A, et al. Patient-specific bioinks for 3D bioprinting of tissue engineering scaffolds. Adv Healthc Mater. 2018 Jun;7(11):1701347. doi: 10.1002/ adhm.201701347

8. Saidy NT, Shabab T, Bas O, Rojas-González DM, Menne M, Henry $\mathrm{T}$, et al. Melt electrowriting of complex 3D anatomically relevant scaffolds. Front Bioeng Biotechnol. 2020 Jul 24;8:793. doi: 10.3389/fbioe.2020.00793

9. Mandrycky C, Wang Z, Kim K, Kim DH. 3D bioprinting for engineering complex tissues. Biotechnol Adv. $2016 \mathrm{Jul}$ Aug;34(4):422-34. doi: 10.1016/j.biotechadv.2015.12.011

10. Gopinathan J, Noh I. Recent trends in bioinks for 3D printing. Biomater Res. 2018 Apr 8;22(1):11. doi: 10.1186/ s40824-018-0122-1

11. Koledova Z. 3D cell culture: an introduction. In: 3D Cell Culture (pp. 1-11). New York: Humana Press; 2017.

12. Vladislav PA, Yusef KD, Stanislav PV, Pavel KA, Elizaveta KV, Elizaveta NK, et al. Magnetic levitational bioassembly of 3D tissue construct in space. Sci Adv. 2020 Jul 15;6(29):eaba4174. doi: 10.1126/sciadv.aba4174

13. Parfenov VA, Petrov SV, Pereira FD, Levin AA, Koudan EV, Nezhurina EK, et al. Scaffold-free, label-free, and nozzle-free magnetic levitational bioassembler for rapid formative biofabrication of 3D tissues and organs. Int J Bioprinting. 2020 Jul 28; 6(3):304. doi: 10.18063/ijb. v6i3.304

14. Rothschild LJ. Synthetic biology meets bioprinting: enabling technologies for humans on Mars (and Earth). Biochem Soc Trans. 2016 Aug 15;44(4):1158-64. doi: 10.1042/BST20160067 